\title{
A New Splitting Technique for Solving Nonlinear Equations by an Iterative Scheme
}

\author{
Chein-Shan Liu \\ Correspondence: Center of Excellence for Ocean Engineering, College of Engineering, National Taiwan Ocean Univer- \\ sity, Keelung 202-24, Taiwan
}

Received: June 10, 2020 Accepted: July 3, 2020 Online Published: July 10, 2020

doi:10.5539/jmr.v12n4p40ＵRL: https://doi.org/10.5539/jmr.v12n4p40

\begin{abstract}
Using a new trick, the nonlinear equation is recast to a coupled system consisting of a linear equation and a nonlinear equation. For the latter, with a weight factor we split the nonlinear term into two parts on both sides of the equation. When the two-dimensional nonlinear system is linearized around the iteration point to be a linear system, we can easily solve it and develop a fast convergent iterative scheme to solve nonlinear equations. In order to further enhance the convergence speed, a linear term is added on both sides of the first linear equation, which results to a very powerful iterative scheme with parameter being analyzed by the eigenvalues. The new iterative scheme is proved to be absolutely convergent, and the number of iterations for convergence is estimated. The merits of the present iterative scheme are insensitive to the initial guess of the solution, convergent very fast, and without needing of the differentials of the function.
\end{abstract}

Keywords: nonlinear equation, splitting technique, iterative scheme, accelerating parameter

\section{Introduction}

In this paper, we derive a fast convergent iterative scheme to solve a scalar nonlinear equation:

$$
f(x)=0,
$$

where we do not need the differential of $f$, which means that $f$ is allowed to be a non-differentiable function of $x$.

The requirement of a solution of Equation (1) is popular and frequently encountered in the applications of engineering and science. For instance, we have to solve

$$
\cos x \cosh x+1=0
$$

for the determination of the natural frequencies of the free vibration of a uniform cantilever beam [Liu \& $\mathrm{Li}$ (2017)]. Equation (2) is a transcendental equation and the closed-form solution does not exist. Indeed, for most nonlinear equations, merely the numerical methods can help us to find the approximate solutions.

Given an initial guess $x_{0}$, from Equation (1) follows an iterative Newton method (NM):

$$
x_{k+1}=x_{k}-\frac{f\left(x_{k}\right)}{f^{\prime}\left(x_{k}\right)}, k=0,1, \ldots,
$$

which is known to be quadratic convergence. Nevertheless, it is well known that the NM is sensitive to the initial guess and may confront a dangerous situation in the fraction term when $f^{\prime}\left(x_{k}\right)$ tends to zero, and in this case the NM will be divergent.

Since the pioneering work of Newton, there are different modifications of the Newton method for more quickly and stably solving nonlinear equations, to name a few [Abbasbandy(2003); Chun (2005); Chun \& Ham (2008); Weerakoon \& Fernando (2000); Yun (2008)]. The homotopy perturbation methods were developed to solve nonlinear equations [Abbasbandy (2006); He (2006); Hesameddini \& Latifizadeh (2009)]. Noor et al. (2010b) have reviewed some multi-step iterative methods for solving nonlinear equations.

\section{A New Splitting Technique}

Without losing any generality, $f(x)$ in Equation (1) can be decomposed to

$$
f(x)=f_{1}(x) x+a x-b,
$$

where $f_{1}(x) x$ is the nonlinear part of $f$, while $a x-b$ is the linear part of $f$.

Let

$$
y:=f_{1}(x) x
$$


be a new variable. Then, from Equations (1), (4) and (5) it follows that

$$
\begin{aligned}
& a x+y=b, \\
& f_{1}(x) x-y=0,
\end{aligned}
$$

which are coupled system of a linear equation and a nonlinear equation for $(x, y)$. The intersection point of the straight line (6) and the curve (7) is just the solution of Equation (1).

For Equation (7), we propose a new splitting technique by

$$
(1-w) f_{1}(x) x-y=-w f_{1}(x) x,
$$

where $w$ is a weight parameter, which will be discussed below.

Next, we develop an iterative scheme to solve Equations (6) and (8), when we know $x_{k}$ at the $k$ th step:

$$
\begin{aligned}
& a x+y=b, \\
& (1-w) f_{1}\left(x_{k}\right) x-y=-w f_{1}\left(x_{k}\right) x_{k},
\end{aligned}
$$

where we have linearized Equation (8) around $x_{k}$, such that Equations (9) and (10) are linear system for $(x, y)$. In doing so, we can seek the next $x_{k+1}$ by solving

$$
\left[\begin{array}{cc}
a & 1 \\
(1-w) f_{1}\left(x_{k}\right) & -1
\end{array}\right]\left[\begin{array}{l}
x \\
y
\end{array}\right]=\left[\begin{array}{c}
b \\
-w f_{1}\left(x_{k}\right) x_{k}
\end{array}\right]
$$

which readily leads to

$$
x_{k+1}=\frac{b-w f_{1}\left(x_{k}\right) x_{k}}{a+(1-w) f_{1}\left(x_{k}\right)} .
$$

Up to here, we no more need the variable $y$, because the iterative scheme (12) is self-contained for $x$.

Multiplying the denominator and nominator on the right-hand side in Equation (12) by $x_{k}$ and using

$$
f_{1}\left(x_{k}\right) x_{k}=f\left(x_{k}\right)-a x_{k}+b
$$

obtained from Equation (4), we can further refine Equation (12) to

$$
x_{k+1}=\frac{(1-w) b x_{k}-w x_{k}\left[f\left(x_{k}\right)-a x_{k}\right]}{a w x_{k}+(1-w)\left[f\left(x_{k}\right)+b\right]}=x_{k}-\frac{x_{k} f\left(x_{k}\right)}{a w x_{k}+b(1-w)+(1-w) f\left(x_{k}\right)},
$$

which is a new iterative scheme to solve Equation (1).

If $a=0$, Equations (6) and (7) return to the original nonlinear Equation (1), and meanwhile Equation (13) is not workable, when $f\left(x_{k}\right)$ tends to $-b$, leading to the divergence of the iterative scheme, or when also $b=0$, yielding an incorrect iteration $x_{k+1}=w x_{k} /(w-1)$. To remedy this shortcoming, we can add a term $c x$ on both the sides of Equation (6), which renders a modification of Equation (13) to

$$
\begin{aligned}
& x_{k+1}=\frac{(1-w) b x_{k}-w x_{k}\left[f\left(x_{k}\right)-a x_{k}\right]+c x_{k}^{2}}{a w x_{k}+(1-w)\left[f\left(x_{k}\right)+b\right]+c x_{k}} \\
& =x_{k}-\frac{x_{k} f\left(x_{k}\right)}{a w x_{k}+b(1-w)+c x_{k}+(1-w) f\left(x_{k}\right)},
\end{aligned}
$$

where $c$ is a given parameter. Obviously, the above iterative scheme satisfies the basic requirement that $x_{k+1} \longrightarrow x_{k}$ when $f\left(x_{k}\right) \longrightarrow 0$. Later, from the analysis of eigenvalues we will examine the advantage obtained by supplementing $c x$ on both sides, even though $a$ is not zero.

It is interesting that the present iterative scheme (14) does not need the differential terms $f^{\prime}\left(x_{k}\right)$ and $f^{\prime \prime}\left(x_{k}\right)$, which are indispensable in many two-step and three-step iterative methods [Noor \& Noor (2006a,2006b); Noor (2007a,2007b); Noor et al. (2006,2010a,2010b); Soheili et al. (2008); Bahgat (2012)].

Remark 1. Since $x=0$ is a fixed point of the iterative schemes (13) and (14), in general we cannot choose $x_{0}=0$ as the initial guess, unless $x=0$ is a trivial solution of $f(x)=0$. The iterative scheme (13) reduces to the fixed point method:

$$
x_{k+1}=x_{k}-f\left(x_{k}\right),
$$


if we take $w=1$ and $a=1$. It is obtained from Equation (1) by

$$
x=x-f(x),
$$

and set the left $x$ to $x_{k+1}$ and the right $x$ to $x_{k}$. In general, the iterative scheme with $w=1$ is not convergent. From this aspect, Equation (8) is clever than the technique in Equation (15), where we split the nonlinear term on both sides of the equation through a weight factor $w$ in Equation (8).

Now, we specify how to choose the parameter $c$, which is acting as another acceleration parameter. As mentioned above we have added a term $c x$ on both the sides of Equation (9), such that Equation (11) is changed to

$$
\left[\begin{array}{cc}
a+c & 1 \\
(1-w) f_{1}\left(x_{k}\right) & -1
\end{array}\right]\left[\begin{array}{l}
x \\
y
\end{array}\right]=\left[\begin{array}{c}
b+c x_{k} \\
-w f_{1}\left(x_{k}\right) x_{k}
\end{array}\right] .
$$

The eigenvalues of the coefficient matrix decide the convergence behavior and its speed. Through some operations, we can find two eigenvalues:

$$
\begin{aligned}
& \lambda_{1}=\frac{1}{2}\left[a+c-1+\sqrt{(a+c-1)^{2}+4\left[a+c+(1-w) f_{1}\left(x_{k}\right)\right]}\right] \\
& \lambda_{2}=\frac{1}{2}\left[a+c-1-\sqrt{(a+c-1)^{2}+4\left[a+c+(1-w) f_{1}\left(x_{k}\right)\right]}\right] .
\end{aligned}
$$

Since we need to inverting the coefficient matrix to obtain the iterative scheme (14), for the convergence of the iterative scheme, the values of $\lambda_{1}$ and $\lambda_{2}$ must satisfy

$$
\left|\lambda_{1}\right|>1,\left|\lambda_{2}\right|>1 \text {. }
$$

For the special case with $w=1$, we have $\lambda_{1}=c$ and $\lambda_{2}=-1$, which is likely to be divergent, depending on the initial value $x_{0}$ and $c$.

Especially, the number of iterations is greatly influenced by $\lambda_{1}$, wherein $\left|\lambda_{2}\right|>1$ and $\lambda_{2}$ is closed to -1 , when $c>0$. Therefore, we have the following estimation of the number of iterations for the convergence:

$$
N=\frac{-\ln \varepsilon}{\ln \left|\lambda_{1}\right|}
$$

where $\varepsilon$ is the tolerance for the error of the solution. How to selecting a suitable value of $c$ is a trade-off between the convergence behavior and the accuracy. Larger value of $c$ will increase the absolute values of $\lambda_{1}$ and $\lambda_{2}$, as a consequence, enhancing the convergence speed; however, it also makes a loss of the accuracy, due to the operation by adding $c x$ on both sides of Equation (9), which, in order to achieve the same convergence criterion, might need more iterations.

\section{Numerical Tests}

In this section, we give some examples to assess the performance of the newly proposed iterative scheme and analyze its mechanism. For some cases we will compare the computed results to the Newton method (NM) and other methods with the same convergence criteria:

$$
\left|x_{k+1}-x_{k}\right|<\varepsilon \text { and }\left|f\left(x_{k+1}\right)\right|<\varepsilon \text {. }
$$

We fix $\varepsilon=10^{-15}$ for all tests, and the maximum number of iterations is set to be 500. Sometimes the NM may lead to spurious solution, satisfying the first criterion but not the second criterion. In order to get rid of the spurious solution, both two criteria are used here.

Example 1. Consider

$$
f(x)=\exp \left(x^{2}+7 x-30\right)-1=0,
$$

which has two solutions $x=-10$ and $x=3$. Here, $a=0$ and $b=1$.

Given $x_{0}=4$, the NM converges with 20 iterations to achieve the solution $x=3$, while the method of Bahgat (2012), shortened as BM, is convergent with 8 iterations. However, when we take $x_{0}<2.6$, both the NM and the BM are divergent. With $x_{0}=2.6$, the NM is convergent with 314 iterations, while the BM is divergent. The present method with $w=-8$ and $c=10$ is convergent with 10 iterations. In this case, $\lambda_{1}=10.27$ and $\lambda_{2}=-1.27$. According to Equation (20), the number $N$ for the convergence is about $N=15$. In practice, the number 10 of iterations is slightly faster than the estimated one.

We list the maximum error (ME) to satisfy $f=0$ with the root $x=3$ and the number of iterations (NI) for different values of $w$ and $x_{0}$ in Table 1 . 
Table 1. For example 1 with the solution $x=3$, comparing the maximum error (ME) and the number of iterations (NI) for different value of $w$ used in the present method with $c=10$

\begin{tabular}{|l|l|l|l|l|l|}
\hline$w, x_{0}$ & $-8,4$ & $-7,4$ & 1,4 & $-5,2$ & $-8,3.5$ \\
\hline ME & 0 & 0 & $\times$ & 0 & 0 \\
\hline NI & 10 & 13 & blow up & 24 & 8 \\
\hline
\end{tabular}

If we take $c=0.1, \lambda_{1}=0.117$, consequently the present method is divergent. If we take $w=1$ and $c=13.4, \lambda_{1}=13.5$ and $\lambda_{2}=-1$, which makes the present method divergence with $x_{0}=4$, and convergence with 39 iterations with $x_{0}=1$. Basically, $w=1$ is not suggested.

For $f=0$ with the root $x=-10$, we list the NI for different values of $w$ and $x_{0}$ in Table 2, which are compared to the NM and the BM.

Table 2. For example 1 with the solution $x=-10$, comparing the maximum error (ME) and the number of iterations (NI) for different value of $w$ used in the present method with $c=-10$

\begin{tabular}{|l|l|l|l|l|l|}
\hline$w, x_{0}$ & $-2,-15$ & $-2,-20$ & $-8,-25$ & $1,-25$ & $-2,-30$ \\
\hline NI of NM & 96 & 236 & 427 & 427 & blow up \\
\hline NI of BM & 34 & blow up & blow up & blow up & blow up \\
\hline NI of present method & 2 & 35 & 31 & blow up & 36 \\
\hline
\end{tabular}

Tables 1 and 2 show that the NI of the present method is quite uniform. With $w=1$, the present method is not applicable. The value of $w$ with $w<1$ is suggested. From these discussions we can observe that the present method is insensitive to the initial value, but both the NM and the BM are sensitive to the initial value.

Remark 2. From Equations (17) and (18), we can observe that when $c$ is given and fixed, we can give more negative value of $w$ to increase the absolute values of $\lambda_{1}$ and $\lambda_{2}$. This is a simple strategy to choose the value of $w$. For example, instead of $w=-2$ in Table 2, we can take $w=-5$, then the INs from 35 and 36 reduce to 29. In Section 4, we will derive a more delicate method to determine $w$, which is allowed to be a function of $x$.

Example 2. In order to accelerate the convergence of the present method, we can also consider a suitable value of $c$, even though $a \neq 0$. Let us consider

$$
f(x)=\exp \left(x^{2}+7 x-30\right)+x+9=0,
$$

where $x=-10$ is a solution. Here, $a=1$ and $b=-9$.

Let $x_{0}=-20$. It is interesting that the present method with $w=-1$ and $c=-20$ is convergent very fast with two iterations to obtain the solution $x=-10$, while the NM converges with 237 iterations and the BM is divergent.

If we take the initial value to be $x_{0}=10$, the three iterative schemes all obtained another solution $x=-9.00000614462766$ of Equation (23), with respectively, 9 iterations for the present method with $c=0,144$ iterations for the NM, and 51 iterations for the BM.

Example 3. Consider

$$
f(x)=\exp (x-1)+x^{4}+x^{3}+x-4=0,
$$

where $x=1$ is a solution. Here, $a=1$ and $b=4$ in the present problem.

Let $x_{0}=0.5$. The present method with $w=-3$ and $c=0$ is convergent with 30 iterations, while the NM converges with 8 iterations. It is amazing that when we take $c=-1$, the present method with $w=-2$ is convergent with 6 iterations, slightly faster than the NM.

Example 4. Let us consider

$$
f(x)=\cos x \cosh x+1=0,
$$

where $x=1.875104068711961$ is a solution. We take $a=0, b=-1, w=0.9$ and $c=-4.1$ in the present method.

In Table 3, we list the NI and the ME for different values of $x_{0}$, which are compared to the NM and the BM. It can be seen that the present method is convergent very fast and is also insensitive to the initial values of $x_{0}$. 

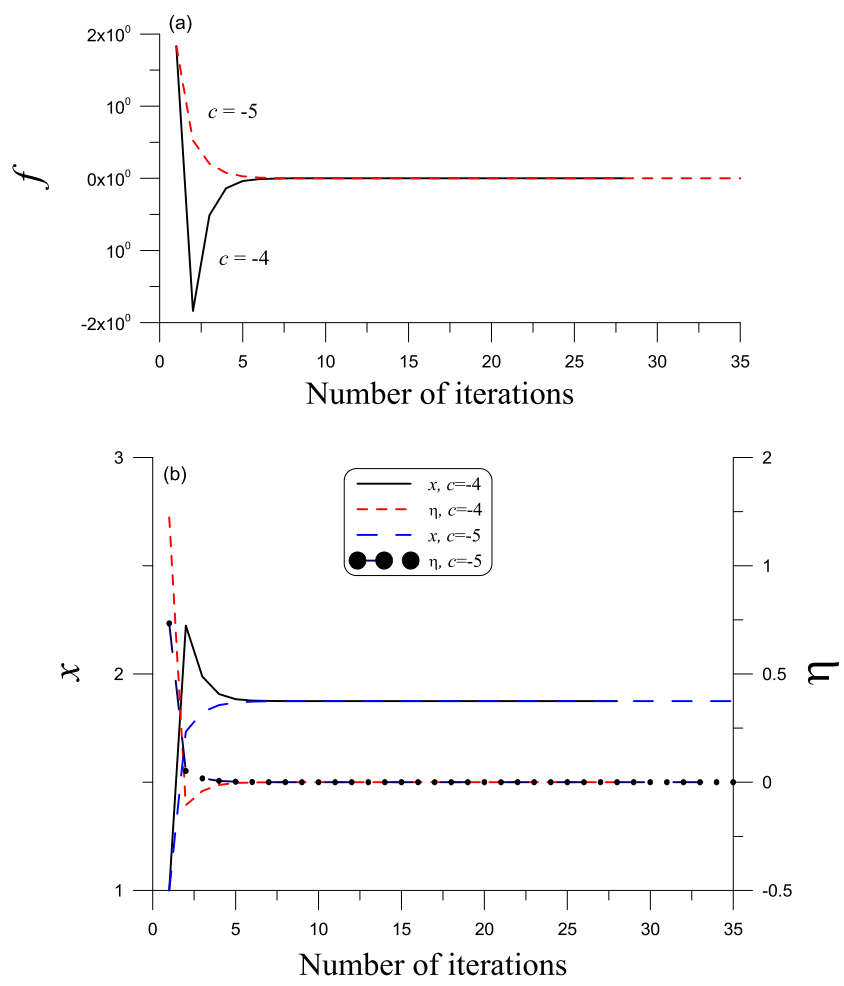

Figure 1. For example 4, (a) the residuals, and (b) the orbits of $x$ and comparing $\eta$ with $c=-4$ and $c=-5$

Table 3. For example 4, comparing the ME and the NI for different value of $x_{0}$, with 500 the maximum number of iterations

\begin{tabular}{|l|l|l|l|l|}
\hline$x_{0}$ & 0.5 & 1.0 & 1.5 & 5.0 \\
\hline NI of NM, ME & $500,1.34 \times 10^{-5}$ & $500,3971544.98$ & $6,1.55 \times 10^{-15}$ & $500,2.37 \times 10^{-14}$ \\
\hline NI of BM, ME & $500,1.34 \times 10^{-5}$ & $6,2.22 \times 10^{-16}$ & $4,6.66 \times 10^{-16}$ & $500,2.37 \times 10^{-14}$ \\
\hline NI of present method, ME & $11,2.22 \times 10^{-16}$ & $10,2.22 \times 10^{-16}$ & $9,1.11 \times 10^{-15}$ & $17,6.66 \times 10^{-16}$ \\
\hline
\end{tabular}

In order to demonstrate the convergent mechanism of the present method, we consider two cases (a) $c=-4$ and (b) $c=-5$, with the same $w=-2$ and $x_{0}=1$. For the case (a), the present method converges with 28 iterations as shown by the solid line in Fig. 1(a), while for the case (b), the present method converges with 35 iterations as shown by the dashed line in Fig. 1(a). After the initial point at the first step, both the residual curves of $f$ are monotonically convergent to zero. At the same time, the orbits of $x$, as shown in Fig. 1(b), are monotonically convergent to the true solution $r=1.875104068711961$.

Upon letting

$$
x_{k+1}=x_{k}\left(1+\eta_{k}\right)
$$

and from Equation (14), we can derive

$$
\eta_{k}=\frac{-f\left(x_{k}\right)}{a w x_{k}+b(1-w)+c x_{k}+(1-w) f\left(x_{k}\right)} .
$$

We plot $\eta_{k}$ in Fig. 1(b), where we can observe two mechanisms to approach to the solution:

$$
\begin{aligned}
& \text { Case (a): }-1<\eta_{k}<\eta_{k+1}<0, f\left(x_{k}\right)<0, x_{k}>x_{k+1}>r, k \geq 2, \\
& \text { Case (b): } 0<\eta_{k+1}<\eta_{k}<1, f\left(x_{k}\right)>0, x_{k}<x_{k+1}<r, k \geq 2 .
\end{aligned}
$$

They indicate that $x_{k}$ is a monotonically decreasing sequence for the Case (a), while for the Case (b), a monotonically increasing sequence. Now, we are in a good position to prove the following result.

Theorem 1. The iterative scheme in Equation (14) for solving $f(x)=0$ is absolutely convergent, for some suitable values of the parameters $w$ and $c$. 
Proof. Let $r$ be a true solution of $f(x)=0$, i.e., $f(r)=0$. We define

$$
e_{k}=x_{k}-r
$$

and it follows that

$$
e_{k+1}-e_{k}=x_{k+1}-x_{k} \text {. }
$$

With a suitable choice of the values of $w$ and $c$, Equations (??) and (??) are easily proved from Equation (??). Without losing generality, we assume that the sequence of $x_{k}$ is $x_{k}>0$ and the real root $r>0$.

We consider the Case (a). From Equations (??) and (??) it follows that

$$
x_{k+1}-x_{k}=x_{k}-x_{k-1}+\eta_{k} x_{k}-\eta_{k-1} x_{k-1}>x_{k}-x_{k-1}+\eta_{k} x_{k}-\eta_{k} x_{k-1}
$$

since $0>\eta_{k}>\eta_{k-1}$ and $x_{k-1}>0$. Then we have

$$
x_{k}-x_{k+1}<\left(1+\eta_{k}\right)\left(x_{k-1}-x_{k}\right) \text {. }
$$

From Equation (??) it follows that

$$
e_{k}-e_{k+1}<\left(1+\eta_{k}\right)\left(e_{k-1}-e_{k}\right) .
$$

Applying this inequality $N-1$ times, we can derive

$$
e_{N}-e_{N+1}<\left(1+\eta_{N}\right) \cdots\left(1+\eta_{2}\right)\left(e_{1}-e_{2}\right) .
$$

Due to $1+\eta_{i}<1, i=2, \ldots, N$, the left sequence converges very fast to zero; hence, $e_{N+1}=e_{N}$ implies $x_{N+1}=x_{N}$. As a consequence, the iterative scheme is absolutely convergent for this case.

Then, we consider the Case (b). From Equation (??), we have

$$
x_{k+1}-x_{k}=\eta_{k} x_{k}
$$

Since the sequence $x_{k}$ has an upper bound with $x_{k}<r$, we merely require to prove that $x_{k}$ is a strictly increasing sequence, that is $x_{k+1}>x_{k}$. Due to $x_{k}>0$, we need to prove that $\eta_{k}>0$. In Equation (??), $f\left(x_{k}\right)>0$, thus we need to prove

$$
a w x_{k}+(1-w)\left[b+f\left(x_{k}\right)\right]+c x_{k}<0,
$$

which can be achieved by selecting $c$ such that

$$
c<-a w+(w-1) \frac{b+f\left(x_{k}\right)}{x_{k}}<0,
$$

where $w<1$. Taking $x_{0}$ into the right-hand side with $c=-a w+(w-1)\left[b+f\left(x_{0}\right)\right] / x_{0}-1$ can hold the above inequality. The sequence $x_{k}$ converges very fast to the true solution $x=r$. Hence, the iterative scheme is absolutely convergent for the Case (b).

\section{Function of $w(x)$}

From the derivations in Section 2, we can observe that $w$ can be a function of $x$ in Equation (8). For the determination of $w(x)$, we let $r$ be a simple solution of $f(x)$, i.e., $f(r)=0$ and $f^{\prime}(r) \neq 0$. We suppose that after $N_{0}$ iterations of Equation (14), the iterative scheme provides a sufficiently close solution to the exact one $r$, and we define $e_{k}=x_{k}-r$. Then, by using the Taylor series we have

$$
f\left(x_{k}\right)=\epsilon=f^{\prime}(r)\left[c_{1} e_{k}+c_{2} e_{k}^{2}+\cdots\right], c_{k}:=\frac{1}{k !} \frac{f^{(k)}(r)}{f^{\prime}(r)}, k=1, \ldots
$$

Let

$$
x_{k+1}=\frac{(1-w) b x_{k}-w x_{k}\left[f\left(x_{k}\right)-a x_{k}\right]+c x_{k}^{2}}{a w x_{k}+(1-w)\left[f\left(x_{k}\right)+b\right]+c x_{k}}=x_{k}(1+\eta),
$$

and we hope $\eta$ to be a small quantity. Some operations lead to

$$
\eta=\frac{-\epsilon}{a w x_{k}+(1-w) \epsilon+(1-w) b+c x_{k}} .
$$


To require an $n$-order convergence rate, we take

$$
\eta=\frac{-\epsilon}{a w x_{k}+(1-w) \epsilon+(1-w) b+c x_{k}}=\frac{-\epsilon}{\epsilon^{1-n}}=-\epsilon^{n} .
$$

Therefore, we can derive

$$
a w x_{k} \epsilon^{n-1}+(1-w) \epsilon^{n}+(1-w) b \epsilon^{n-1}+c x_{k} \epsilon^{n-1}=1,
$$

which generates

$$
w=\frac{1-b \epsilon^{n-1}-\epsilon^{n}-c x_{k} \epsilon^{n-1}}{a x_{k} \epsilon^{n-1}-\epsilon^{n}-b \epsilon^{n-1}} .
$$

Hence, $w$ may abide the following rule:

$$
w=\left\{\begin{array}{l}
w_{0}, \quad k \leq N_{0}, \\
\frac{1-b \epsilon^{n-1}-\epsilon^{n}-c x_{k} \epsilon^{n-1}}{a x_{k} \epsilon^{n-1}-\epsilon^{n}-b \epsilon^{n-1}}, k>N_{0} .
\end{array}\right.
$$

If we take $N_{0}=500$ to be the maximum number of iterations, we return to the original iterative scheme. If we take $N_{0}=0$, then we fully use the new technique in the iterative scheme. Otherwise, it is a mixed type iterative scheme.

For example 3, we apply this modified method with $c=-2, x_{0}=0.5, w_{0}=-2$ and $n=3$, and we list the NI for different values of $\epsilon$ and $N_{0}$ in Table 4 .

Table 4. For example 3 with the solution $x=1$, comparing the number of iterations (NI) for different value of $\epsilon$ and $N_{0}$ used in the present method

\begin{tabular}{|l|l|l|l|l|l|}
\hline$N_{0}, \epsilon$ & $500,0.315$ & $10,0.315$ & $5,0.315$ & $0,0.315$ & $0,0.300$ \\
\hline NI of present method & 18 & 14 & 13 & 10 & 18 \\
\hline
\end{tabular}

It can be seen that the new strategy is effective, and it is sensitive to the value of $\epsilon$. The original iterative scheme needs 18 iterations, while the new one with $N_{0}=0$ and $\epsilon=0.315$ needs 10 iterations.

In order to further compare the iterative scheme (14) to other methods, we consider the following test functions:

$$
\begin{aligned}
& f_{1}(x)=\exp \left(x^{2}+7 x-30\right)-1, \\
& f_{2}(x)=\sin ^{2} x-x^{2}+1, \\
& f_{3}(x)=x^{2}-e^{x}-3 x+2, \\
& f_{4}(x)=(x-1)^{3}-1, \\
& f_{5}(x)=x^{3}-10 .
\end{aligned}
$$

In Table 5, for different functions we list the NI obtained by the present algorithm (14), which are compared to the NM, the method of Noor et al. (2006) (NNT), the method of Chun (2005) (CM), and the method of Noor et al. (2010b) (NRM).

Table 5. The comparison of different methods for the number of iterations

\begin{tabular}{|l|l|l|l|l|l|l|}
\hline Functions & $x_{0}$ & NM & NNT & CM & NRM & Present \\
\hline$f_{1}$ & 3.5 & 13 & 7 & 8 & 7 & 7 \\
\hline$f_{2}$ & -1 & 7 & 5 & 5 & 4 & 8 \\
\hline$f_{3}$ & 2 & 6 & 5 & 4 & 4 & 5 \\
\hline$f_{4}$ & 3.5 & 8 & 5 & 5 & 5 & 6 \\
\hline$f_{5}$ & -2 & 7 & 5 & 5 & 4 & 4 \\
\hline
\end{tabular}




\section{Conclusions}

Employing the new trick of a new idea, the nonlinear equation was recast to a coupled system of a linear equation and a nonlinear equation. With a weight factor $w$, we split the nonlinear term into two parts on both sides of the equation. Through the consideration of the eigenvalues, a linear term $c x$ was also added on both sides of the first linear equation. Then, we can easily derive the iterative scheme by solving the linearized system. The iterative scheme was proven to be absolutely convergent, and the iterations' number for the convergence was analyzed and provided by using the eigenvalues. Upon comparing four examples to other methods, we found that the present iterative scheme possessed three major advantages: insensitive to the initial value, convergent very fast, and without needing of the differentials of the function. Further tests on five functions revealed that the present iterative scheme was competitive to the cubic convergence iterative scheme, as well as to the fourth-order convergence iterative scheme.

\section{References}

Abbasbandy, S. (2003). Improving Newton-Raphson method for nonlinear equations by modified Adomian decomposition method. Appl. Math. Comput., 145, 887-893. https://doi.org/10.1016/S0096-3003(03)00282-0

Abbasbandy, S. (2006). Modified homotopy perturbation method for nonlinear equations and comparison with Adomian decomposition method. Appl. Math. Comput., 172, 431-438. https://doi.org/10.1016/j.amc.2005.02.015

Bahgat, M. S. M. (2012). New two-step iterative methods for solving nonlinear equations. J. Math. Res., 4, 128-131. https://doi:10.5539/jmr.v4n3p128

Chun, C. (2005). Iterative methods improving Newton's method by the decomposition method. Comput. Math. Appl., 50, 1559-1568. https://doi.org/10.1016/j.camwa.2005.08.022

Chun, C., \& Ham, Y. (2008). Some fourth-order modifications of Newtons method. Appl. Math. Comput., 197, 654-658. https://doi.org/10.1016/j.amc.2007.08.003

He, J. H. (2006). Some asymptotic methods for strongly nonlinear equations. Int. J. Modern Phys. B, 20, 1141-1199. https://doi.org/10.1142/S0217979206033796

Hesameddini, E., \& Latifizadeh, H. (2009). An optimal choice of initial solutions in the homotopy perturbation method. Int. J. Nonl. Sci. Numer. Simul., 10, 1389-1398. https://doi.org/10.1515/IJNSNS.2009.10.11-12.1389

Liu, C.-S., \& Li, B. (2017). An upper bound theory to approximate the natural frequencies and parameters identification of composite beams. Compos. Struct., 171, 131-144. https://doi.org/10.1016/j.compstruct.2017.03.014

Noor, M. A., \& Noor, K. I. (2006a). Three-step iterative methods for nonlinear equations. Appl. Math. Comput., 183, 322-327. https://doi.org/10.1016/j.amc.2006.05.055

Noor, M. A., \& Noor, K. I. (2006b). Some iterative schemes for nonlinear equations. Appl. Math. Comput., 183, 774-779. https://doi.org/10.1016/j.amc.2006.05.084

Noor, M. A., Noor, K. I., Mohyud-Din, S. T., \& Shabbir, A. (2006) An iterative method with cubic convergence for nonlinear equations. Appl. Math. Comput., 183, 1249-1255. https://doi.org/10.1016/j.amc.2006.05.133

Noor, K. I. (2007a). New iterative schemes for nonlinear equations. Appl. Math. Comput., 187, 937-943. https://doi.org/10.1016/j.amc.2006.09.028

Noor, K. I. (2007b). New family of iterative methods for nonlinear equations. Appl. Math. Comput., 190, 553-558. https://doi.org/10.1016/j.amc.2007.01.045

Noor, K. I., \& Noor, M. A. (2007). Predictor-corrector Halley method for nonlinear equations. Appl. Math. Comput., 188, 1587-1591. https://doi.org/10.1016/j.amc.2006.11.023

Noor, M. A., Noor, K. I., \& Waseem, M. (2010a). Fourth-order iterative methods for solving nonlinear equations. Int. J. Appl. Math. Eng. Sci., 4, 43-52. https://doi.org/10.1016/j.amc.2006.11.023

Noor, M. A., Noor, K. I., Al-Said, E., \& Waseem, M. (2010b). Some new iterative methods for nonlinear equations. Math. Prob. Eng., 2010, ID:198943. https://doi.org/10.1155/2010/198943

Soheili, A. R., Ahmadian, S. A., \& Naghipoor, J. (2008). A family of predictor-corrector methods based on weight combination of quadratures for solving nonlinear equations. Int. J. Nonl. Sci., 6, 29-33. https://doi.org/IJNS.2008.08.15/159

Weerakoon, S., \& Fernando, T. G. I. (2000). A variant of Newtons method with accelerated third-order convergence. Appl. Math. Lett., 13, 87-93. https://www.elsevier.nl/locate/aml 
Yun, J. H. (2008). A note on three-step iterative method for nonlinear equations. Appl. Math. Comput., 202, 401-405. https://doi.org/10.1016/j.amc.2008.02.002

\section{Copyrights}

Copyright for this article is retained by the author(s), with first publication rights granted to the journal.

This is an open-access article distributed under the terms and conditions of the Creative Commons Attribution license (http://creativecommons.org/licenses/by/4.0/). 